Tropical Journal of Pharmaceutical Research February 2020; 19 (2): 361-369

ISSN: $1596-5996$ (print); 1596-9827 (electronic)

(C) Pharmacotherapy Group, Faculty of Pharmacy, University of Benin, Benin City, 300001 Nigeria.

Available online at http://www.tjpr.org

Original Research Article

http://dx.doi.org/10.4314/tjpr.v19i2.19

\title{
New insight into single phase formation of capric acid/menthol eutectic mixtures by Fourier-transform infrared spectroscopy and differential scanning calorimetry
}

\author{
Hiba H Ali ${ }^{1}$, Mowafaq M Ghareeb ${ }^{2}$, Mayyas Al-Remawi ${ }^{3}$, Faisal T Al-Akayleh ${ }^{3 *}$ \\ ${ }^{1}$ Department of Pharmaceutics, College of Pharmacy, University of Sulaimani, Sulaimani, Kurdistan Region, ${ }^{2}$ College of \\ Pharmacy, University of Baghdad, Baghdad, Iraq, ${ }^{3}$ Department of Pharmaceutics and Pharmaceutical Technology, Faculty of \\ Pharmacy, University of Petra, Amman, Jordan
}

*For correspondence: Email: falakayleh@uop.edu.jo

Sent for review: 22 May 2019

Revised accepted: 25 January 2020

\begin{abstract}
Purpose: To examine the structural changes of a eutectic mixture comprising capric acid and menthol which are commonly used in pharmaceutical applications.

Methods: A phase diagram was constructed by quantitative mixing of capric acid and menthol under controlled conditions until a single liquid phase was formed. Eutectic mixtures of capric acid: menthol at the ratios of 3:2, 1:4, 1:1, 2:3, and 1:4 were prepared. Hydrogen bond formation and conformational changes were analyzed using Fourier-transform infrared spectroscopy (FTIR) and differential scanning calorimetry (DSC). Microscopic imaging was carried out to capture phase change events upon increasing temperature.

Results: Menthol confirmed the intact structure of a hexagonal ring. The high degree of broadening of the menthol O-H groups indicates hydrogen bond formation. FTIR band changes related to capric acid suggest a break-up of the methylene arrangement structure due to changes in the $\mathrm{C}-\mathrm{H}$ band frequencies. The red shift encountered in $\mathrm{C}=\mathrm{O}$ stretching band emphasizes hydrogen bond formation taking place between the oxygen atom of the hydroxyl group comprising the carboxylic moiety of capric acid and the hydrogen atom of menthol hydroxyl group. DSC results indicate the presence of two polymorphs of the capric acid/ menthol complex. Both exhibited crystallization and conformational change exotherms in addition to two melting endotherms as result of transformation of crystalline components to become partially crystalline due to hydrogen bond formation.

Conclusion: The interaction between capric acid and menthol results in a typical preparation of deep eutectic systems that can act as natural-based solvents in numerous pharmaceutical applications.
\end{abstract}

Keywords: Eutectic system, Capric acid, Menthol, Differential scanning calorimetry, DSC, Fourier transform infrared spectroscopy, FTIR

This is an Open Access article that uses a fund-ing model which does not charge readers or their institutions for access and distributed under the terms of the Creative Commons Attribution License (http://creativecommons.org/licenses/by/4.0) and the Budapest Open Access Initiative (http://www.budapestopenaccessinitiative.org/read), which permit unrestricted use, distribution, and reproduction in any medium, provided the original work is properly credited.

Tropical Journal of Pharmaceutical Research is indexed by Science Citation Index (SciSearch), Scopus, International Pharmaceutical Abstract, Chemical Abstracts, Embase, Index Copernicus, EBSCO, African Index Medicus, JournalSeek, Journal Citation Reports/Science Edition, Directory of Open Access Journals (DOAJ), African Journal Online, Bioline International, Open-J-Gate and Pharmacy Abstracts

\section{INTRODUCTION}

Eutectic systems have emerged as a new outstanding environmental-friendly technique that has presented promising practicalities and applications in the pharmaceutical industry [1,2]. In this context, the theory of phase change materials constitutes the solid platform that 
enables specific solid components to liquidate from their solid state under reasonable process conditions. The formed liquid state was found to be beneficial in increasing drug solubility and thus permeability and/or absorption, all contributing to the increased drug bioavailability in the human body [3]. Different types of organic materials are capable, when mixed together, of forming a eutectic system of a single liquid phase. Amongst these materials, the fatty acidterpene systems have been recently given a considerable interest due to their low toxicity, chemical stability, and biodegradability. Moreover, these systems are characterized by their smaller volume change during phase transition in addition to their low melting temperature. Palmitic acid-camphene [4], pyruvic acid or acetic acid or L-lactic acid or lauric acidmenthol [5], camphor or borneol or N-Ethyl-5methyl-2-(1-methylethyl)

cyclohexanecarboxamide-menthol [6] are good examples of the fatty/ terpene eutectic systems.

The principle of single phase formation for eutectic systems is commonly reported as a basic association between entities comprising electron donor and electron acceptor groups [7]. These groups bind together through hydrogen bond formation between the hydroxyl group $\mathrm{OH}$ of one compound with the carbonyl group $\mathrm{CO}$ - of the other [8]. This pathway of bond formation has been identified through the application of Fourier-transform infrared (FTIR) spectroscopy analysis of the individual components and their eutectic liquid. In this regard, a number of changes in band modes of these two groups- either stretching or bendinghas been assigned to hydrogen bonding. For example, one of the mostly recognized changes associated with this type of bonding was the $\mathrm{C}=\mathrm{O}$ stretching band of eutectic systems [9].

In this context, hydrogen bond formation between the hydroxyl and carbonyl groups was initially considered to take place in the form of an FTIR red shift of the $\mathrm{C}=\mathrm{O}$ stretching band to lower frequencies [10,11]. A typical example of a red shift interaction in fatty acid/ terpene eutectic mixtures was demonstrated by Phaechamud et al [9] when they tested the interaction in a eutectic mixture comprising camphor and menthol. In contrast, the same type of eutectic systems showed no indication of hydrogen bond formation when palmitic acid and camphene eutectic system was tested $[4,12]$. On the other hand, a blue shift to a higher carbonyl wavenumber was reported in the interaction causing a single phase formation when DLmenthol was mixed with either pyruvic acid or acetic acid or L-lactic acid or lauric acid [5].
Differential scanning calorimetry (DSC) was used to highlight the thermal properties of various eutectic systems. Initially, Shilei et al [13] demonstrated no variation in the melting temperatures detected as one single DSC endotherm at different compositions of a eutectic system comprising capric acid and lauric acid. In another eutectic system comprising polyurethane/lauric acid, Kong et al [14] were able to detect two exothermic peaks and one endothermic peak representing the crystallization and melting temperatures respectively. However, identification of these peaks was not discussed thoroughly to highlight any possible polymorphic transformation taking place. With regard to fatty acid-terpene eutectic systems, there is still no sufficient information available for a better understanding of the systems thermal properties. Therefore, the lack of information on DSC thermal properties of the fatty acid-terpene eutectic systems that maybe linked to confusion presented by the FTIR analysis is needed in the current work to be elucidated. Based upon that, the work described herein takes the initiatives to investigate the FTIR and DSC of this type of eutectic system. Capric acid/ menthol system will be given for the first time a great focus to understand their interaction and thermal behavior in the context of novel components of eutectic systems.

\section{EXPERIMENTAL}

\section{Materials}

Capric acid or 1-nonanecarboxylic acid, C10) and (-)-menthol (2-Isopropyl-5-methylcyclohexanol, and menthol were purchased from Merck company (Darmstadt, Germany).

\section{Construction of eutectic phase diagram}

Menthol samples (10-90 g) were added to reagent bottles $(100 \mathrm{~mL})$ in a temperaturecontrolled water bath shaker (Labcompare, CA, USA). Known quantities of capric acid-in the solid state- were then gradually added to menthol until a mixture of liquid and solid phases was formed. Addition was terminated when a single clear phase was formed. All quantities were numerically recorded and added up for all the 9 samples of menthol. The experiment was repeated for the whole temperature range 15 - 35 ${ }^{\circ} \mathrm{C}$. The experiment was repeated three time.

\section{Eutectic mixture preparation}

Capric acid (40 g) and menthol (10 g) were mixed in a reagent bottle $(100 \mathrm{~mL})$ fixed in the shaker which, the later, was adjusted to room 
temperature. Shaking was terminated until a single liquid phase was formed for the 4:1 capric acid menthol mixture. Other capric acid/ menthol ratios; i.e. $3: 2,1: 1,2: 3$, and $1: 4$ were further tested (in triplicate) using the capric acid: menthol amounts (g) of 30:20, 25:25, 20:30, and 10:40 respectively.

\section{FTIR analysis}

Approximate amounts $(\approx 20 \mathrm{~mL}$ or $\approx 20 \mathrm{~g}$ ) of the liquid eutectic mixtures or the solid components were analyzed using FTIR technique (Bruker Optik, Ettlingen, Germany) equipped with a sampling module (QuickSnap ${ }^{\mathrm{TM}}$ ) operating for solid and liquid materials.

\section{DSC analysis}

An approximate weight (5-9 mg) of the eutectic mixture sample was added in a special pan. The foregoing, together with an empty pan (used as a reference) were placed in the DSC instrument (Perkin-Elmer Corporation, Norwalk, CT) whereby the system was set to cool down to -20 ${ }^{\circ} \mathrm{C}$ followed by heating at $10^{\circ} \mathrm{C} / \mathrm{min}$ until melting takes place. The experiment was carried out for all the capric acid: menthol ratios.

\section{Microscopic imaging}

Images of phase changes for the sample containing capric acid: menthol eutectic system at the $1: 1$ mass ratio were captured using Linkam optical microscope (Linkam Scientific Instruments, FDCS196 Freeze drying system, Surrey, UK). Distances of the objective and condenser lenses were set at 4.5 and $12.5 \mathrm{~mm}$ away from the sample respectively. Both lenses were isolated from the sample and the heating/cooling element by a glass Lid/or base window.

\section{RESULTS}

The profile for the binary mixture of menthol/ capric acid mass ratios in relation to temperature represented a typical eutectic phase diagram (Figure 1). The solvents, at all ratios, formed a single liquid phase at temperatures $>20^{\circ} \mathrm{C}$. In other words, room temperature was sufficient for the formation of a clear liquid phase at all mass fractions of menthol and capric acid. However, when the temperature is lower than room temperature, the mixtures were turbid which indicates partial solidification of capric acid and/or menthol. The mixture of both components remained solid below $15^{\circ} \mathrm{C}$.

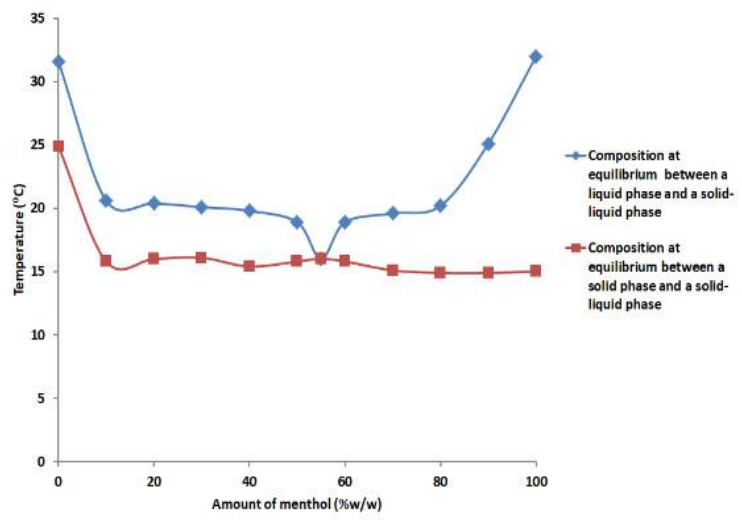

Figure 1: Phase diagram of the eutectic system comprising capric acid and menthol mixtures

The interaction between menthol and capric acid was tested using FTIR and DSC analysis of the mixtures. Initially, the FTIR spectrum of menthol is illustrated in Figure 2 whereby the spectrum shows an $\mathrm{OH}$ stretching at $3260 \mathrm{~cm}^{-1}(\mathrm{H}-$ bonded), $2959 \mathrm{~cm}^{-1}$ and $2870 \mathrm{~cm}^{-1}$ (C-H stretching), and $995 \mathrm{~cm}^{-1}$ and $1226 \mathrm{~cm}^{-1}$ (C-O stretching) $[15,16]$. Bands at $845-974 \mathrm{~cm}^{-1}$ indicate the $\mathrm{C}-\mathrm{C}$ stretching mode of the hexagonal ring. At $1368 \mathrm{~cm}^{-1}$ to $1471 \mathrm{~cm}^{-1}$ frequencies, menthol displayed $\mathrm{C}-\mathrm{H}$ bending typical of methyl groups present within menthol [12]. Bands at 547 and $668 \mathrm{~cm}^{-1}$ are due to the skeletal mode vibrations of the hexagonal ring in the menthol structural unit.

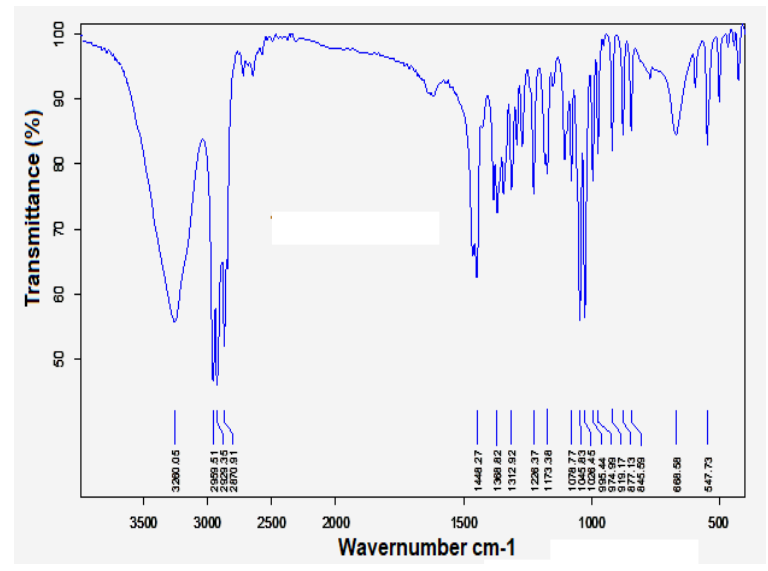

Figure 2: FTIR of menthol

Capric acid presented a typical carboxylic acid compound whereby strong stretching $\mathrm{O}-\mathrm{H}$ and $\mathrm{C}-$ $\mathrm{H}$ bands are displayed at $2851 \mathrm{~cm}^{-1}$ and 2920 $\mathrm{cm}^{-1}$ respectively and another strong $\mathrm{C}=\mathrm{O}$ stretching band of the carbonyl group is displayed at $1699 \mathrm{~cm}^{-1}$ (Figure 3) [3]. In addition, the $\mathrm{O}-\mathrm{H}$ bend modes are typically presented at $1415 \mathrm{~cm}-1$ and $932 \mathrm{~cm}^{-1}$ bands. The last band which manifested a typical carboxylic acid compound is the stretching band of C-O at 1297 $\mathrm{cm}^{-1}$. Other bands are related to the hydrocarbon 
chain whereby the $\mathrm{C}-\mathrm{H}$ bending mode is presented at around $560 \mathrm{~cm}^{-1}$ in addition to 722 $\mathrm{cm}^{-1}$. Moreover, the bands in the range; 932$1231 \mathrm{~cm}^{-1}$ assign the $\mathrm{C}-\mathrm{H}$ stretching mode whereas bands in the range; $1432 \mathrm{~cm}^{-1}$ and 1451 $\mathrm{cm}^{-1}$ represent the $\mathrm{C}-\mathrm{C}$ stretching of the hydrocarbon chain. It has to be noted that there is no appearance of dissociated carboxylate group (COO-) for capric acid at $1575 \mathrm{~cm}^{-1}$ due to its dimeric form [13].

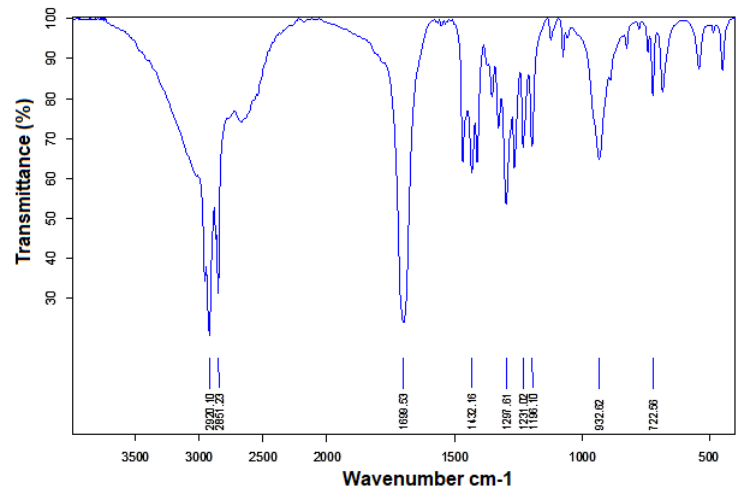

Figure 3: FTIR of capric acid

When the two powders were mixed at the mass ratio of $1: 1 \mathrm{w} / \mathrm{w}$, the main FTIR features of the resulting liquid mixture are presented in Figure 4. Initially, bands broadening between $2652 \mathrm{~cm}^{-1}$ to $2924 \mathrm{~cm}^{-1}$ represents typical structural disorder attributed to the loss in the crystalline structure of molecules. This is due to the phase change that took place when the two solid components were mixed. Similar disorder is noticed at the carbonyl group of capric acid displayed at $1699 \mathrm{~cm}^{-1}$ whereby peak broadening was clearly dominant.

The menthol ring structural encountered no change. Nevertheless, the appearance of new bands with broad transmittance at 1259, 1385, 1422, $1456 \mathrm{~cm}^{-1}$ suggests $\mathrm{C}-\mathrm{H}$ methylene wagging of the capric acid hydrocarbon chain. Such wagging usually arises upon re-orientation of the $\mathrm{H}$ atoms of the methylene groups which commonly takes place upon heating/melting of fatty acids [14]. The collapse of the methylene groups orientation is associated with the motion of the $\mathrm{C}=\mathrm{O}$ and $\mathrm{C}-\mathrm{O}$ electronic cloud which renders changes to their bond length. The foregoing is a typical broadening and shift of the $\mathrm{C}=\mathrm{O}$ band at $1699 \mathrm{~cm}^{-1}$ of capric acid to a higher wavenumber recorded at $1712 \mathrm{~cm}^{-1}$ for the composite mixture. Such changes are suggested to be correlated to the influence of nearby electronic clouds of menthol that are physically in close proximity to the capric acid structural groups.

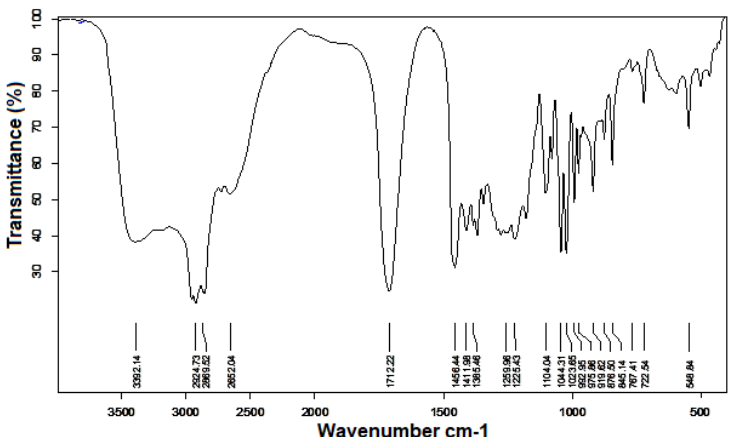

Figure 4: FTIR spectra of the eutectic mixture of capric acid/menthol at the mass ratio of $1: 1$

Frequencies of the $\mathrm{C}-\mathrm{H}$ bending modes of the $\mathrm{CH}_{3}$ groups in menthol at $1368 \mathrm{~cm}^{-1}$ to 1471 $\mathrm{cm}^{-1}$ became highly broader when menthol was present with capric acid. Within this range, it has to be noted that the band at $1471 \mathrm{~cm}^{-1}$ no longer existed. In addition, the strong $\mathrm{O}-\mathrm{H}$ stretching at $3260^{-1}$ for menthol appeared to be spanning in a broader and higher intensity transmittance. This behavior is attributed to the fact that higher hydrogen bonded atoms give rise to higher broadening of the O-H stretching frequency [15].

The DSC technique is another efficient technique to provide an insight into the structural and conformational changes during eutectic system formation. Figure 6 shows a typical DSC thermal profile for menthol and capric acid in the solid state, respectively. The thermograms of both components showed melting endotherms represented by the sharp peaks at 31 and 31.6 ${ }^{\circ} \mathrm{C}$ for menthol and capric acid respectively. With regard to menthol, another two main observations was noticed; menthol existed as one single enantiomer represented by the levorotatory (L-) type and there was no indication of glass transition step by the DSC monograph.

In the former context, the DSC thermogram excluded the presence of the dextrorotatory (D-) type enantiomer which usually shows a melting endotherm at $27{ }^{\circ} \mathrm{C}$ [19]. In other words, the current menthol used herein did not show any thermal profile of racemic mixed structure comprising the DL menthol [20]. In the later context, the absence of the glass transition behavior reflects the highly crystalline structure of menthol or more precisely the absence of amorphous regions which usually undergo glass transition upon heating [21].

As the mixture comprising capric acid and menthol is liquid at room temperature, heating of the mixture was set at $-20^{\circ} \mathrm{C}$ for the temperature controlled DSC instrument. The thermogram of the $1: 1 \mathrm{w} / \mathrm{w}$ capric acid: menthol mixture is 
presented in Figure 7 . The thermal profile of such mixture differs from the single components of capric and menthol as the former presented exothermic, endothermic, and glass transition peaks/regions.

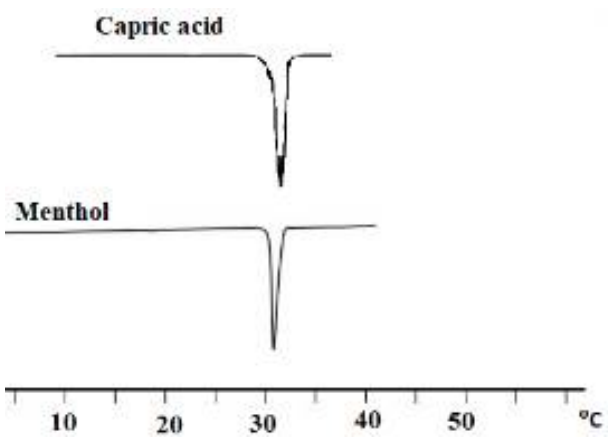

Figure 6: Thermograms of capric acid and menthol in their solid state

Initially, for the DSC region above $0^{\circ} \mathrm{C}$, two broad endothermic/or melting peaks appeared at $3.4^{\circ} \mathrm{C}$ and $15^{\circ} \mathrm{C}$. The first peak more likely represents the melting of a metastable polymorphic form and the second one is related to the capric acid-menthol complex. The melting phase transition endotherm of this complex occurred, as expected, at a lower temperature recorded at $15^{\circ} \mathrm{C}$. However, the peak, as such, appeared much broader than each of the individual melting endotherms for menthol and capric acid. This indicates that chemical or physical interaction has disrupted the crystal lattice of both components.

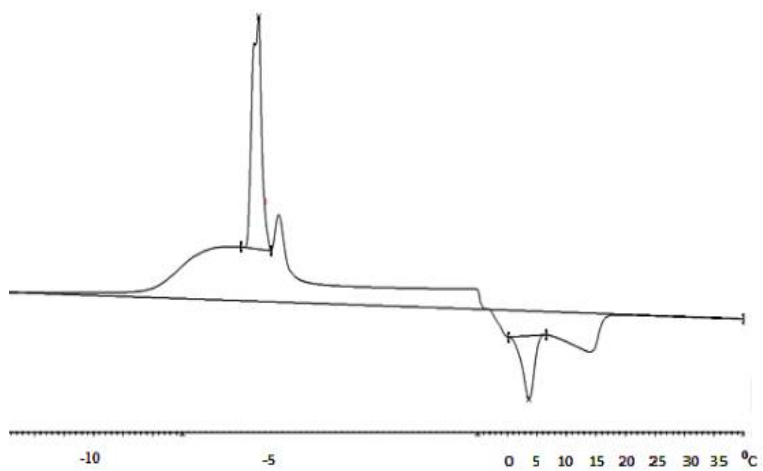

Figure 7: DSC of the eutectic mixture of capric acid: menthol at the mass ratio of $1: 1$

When DSC was performed over another two mass content ratios of capric acid: menthol, i.e. for the $1: 4$ and for the $4: 1$, the thermal profiles are presented in Figure 8 and 9 respectively. Initially, at the 1:4 mass ratio (Figure 8), there was no change to all the peaks presented earlier in Figure 7 (1:1 ratio) except that the second exothermic peak corresponding to a conformational change was equivalent in intensity to the crystallization peak. Moreover, the second melting peak became broader in width. The later peak had a maximum at $25{ }^{\circ} \mathrm{C}$ indicating an increase in the melting temperature although it was still lower than the melting endotherms of the individual components. At the 4:1 mass ratio in Figure 9, the main melting isotherm (second peak) became relatively sharper with higher intensity, the first melting peak became smaller, the second exothermic peak -corresponding to a conformational changeno longer existed and finally the first exothermic peak of crystallization became shaper and higher in intensity.

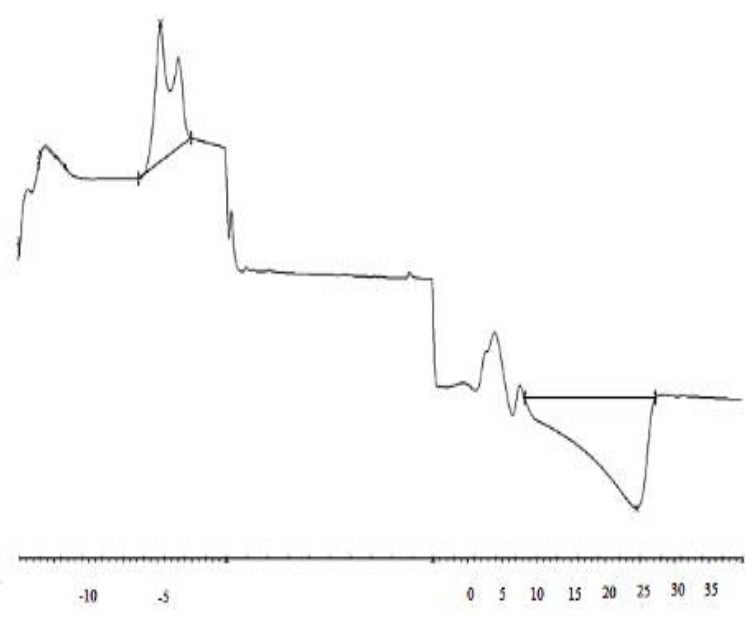

Figure 8: DSC of the eutectic mixture of capric acid: menthol at the mass ratio of 1:4. Note: The unit of measurement of $\mathrm{x}$-axis is ${ }^{\circ} \mathrm{C}$

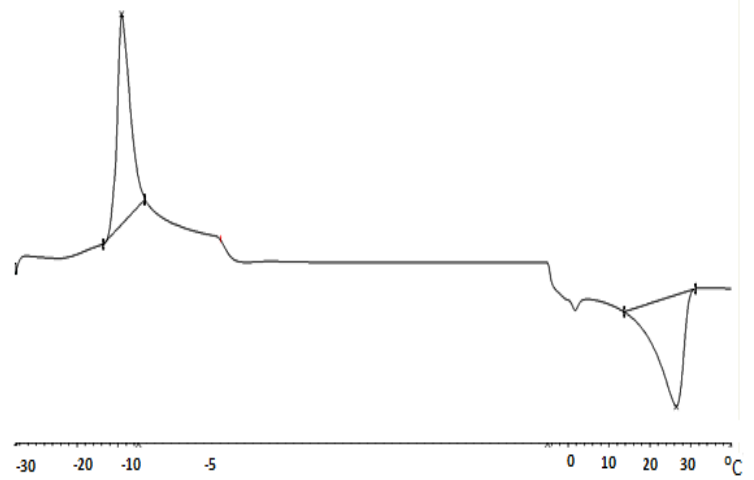

Figure 9: DSC of the eutectic mixture of capric acid: menthol at the mass ratio of $4: 1$.

When the 1:1 capric acid/ menthol system was visually examined under a microscope upon heating the frozen molten from -10 to $25^{\circ} \mathrm{C}$, the captured images are illustrated in Figure 10. Initially, images having a mixed color of black and pink zones indicate the presence of a mixture of solid and liquid phases of the system. Images captured at -7 to $3^{\circ} \mathrm{C}$ had more intensity in black zones than that captured from 5 to 25 
${ }^{\circ} \mathrm{C}$. As stated earlier the first range of temperature $\left(-7{ }^{\circ} \mathrm{C}\right.$ to $\left.3{ }^{\circ} \mathrm{C}\right)$ represents transitional states developed during the course of heating the already solidified particles from glass transition state through crystallization and finally ending with a conformational change. The presence of these solid particles is obviously a result of the interaction between capric acid and menthol. The second range of temperature (5 to $25^{\circ} \mathrm{C}$ ) manifests a decline in the presence of black zones up to a temperature of $17{ }^{\circ} \mathrm{C}$ whereby a complete pink zone had prevailed in the image. However, solid particles at early stages of this range still persisted to exist up to a temperature of $10^{\circ} \mathrm{C}$.
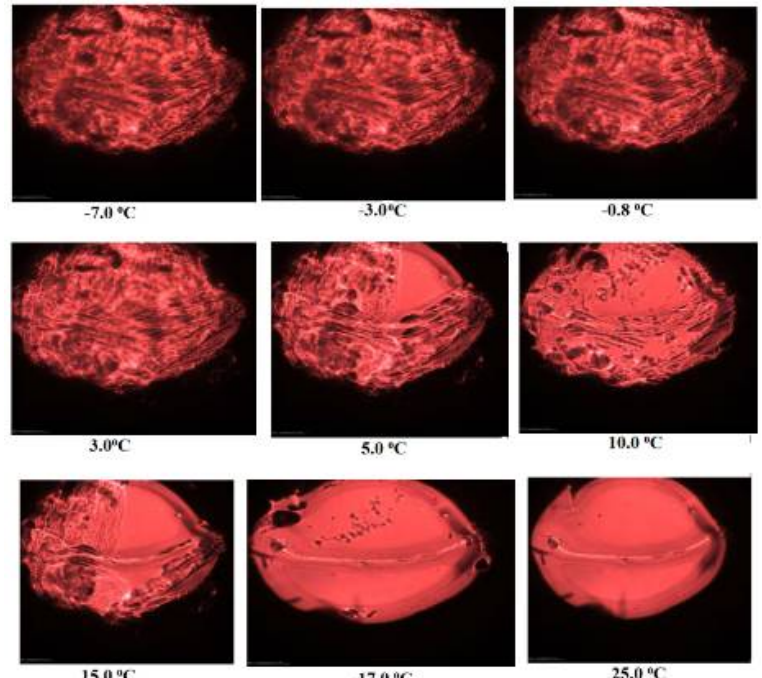

Figure 10: Microscopic images captured upon heating a solidified eutectic mixture of capric acid: menthol at the mass ratio of $1: 1$

\section{DISCUSSION}

The preparation of the menthol/ capric acid mixture (Figure 1) has presented an advantageous eutectic system over other mixtures. Liquidation of the menthol/ capric acid mixture spreads out to a wide range (5 - 90 $\%$ w/w), unlike menthol/ camphor system. Moreover, working at a mass fraction of $55 \%$ $w / w$ menthol can guarantee liquidation of the binary mixture at temperatures around $15{ }^{\circ} \mathrm{C}$. Such liquidation was recorded at $-1{ }^{\circ} \mathrm{C}$ for the menthol/ camphor system [5].

Theoretically, hydrogen bonding represents the major mode of interaction responsible for the formation of a melt phase when a eutectic mixtures is present. The specific atoms involved in bonding are suggested to take place between the hydroxyl group in small molecules and the carbonyl groups in another molecule [6]. Hydrogen bonding in eutectic systems in general results in the red shift of $\mathrm{C}=\mathrm{O}$ stretching band to a lower frequency. This is attributed to bond formation between carbonyl and hydroxyl groups causing lengthening of the $\mathrm{C}=\mathrm{O}$ band [7].

However, in the menthol-capric acid eutectic system, the case was found to follow a different pathway of hydrogen bonding whereby the $\mathrm{C}=\mathrm{O}$ stretching band of capric acid was shifted to a higher frequency. Such shift indicates strengthening and shortening of the band due to absence of a red shift of the $\mathrm{C}=\mathrm{O}$ stretching. The foregoing results from conjugation to the oxygen atom of the carbonyl group [16]. Thus the resonance structure of the carbonyl group was influenced by a nearby bonding which drew its electron density close to the carbon atom of the carbonyl group.

A possible alternative pathway of hydrogen bonding is suggested to have taken place at the oxygen atom of the hydroxyl group comprising the carboxylic moiety of capric acid by the hydrogen atom of menthol hydroxyl group (Figure 5). Such hydrogen bonding is responsible for the red shift of carbonyl group and further for the conformational changes of the capric acid methylene configuration. The foregoing is more likely a cis-isomerisation structural change whereby all methylene groups are on the same side of the carbon [17]. Such isomerization is responsible for lowering the melting point of capric acid.

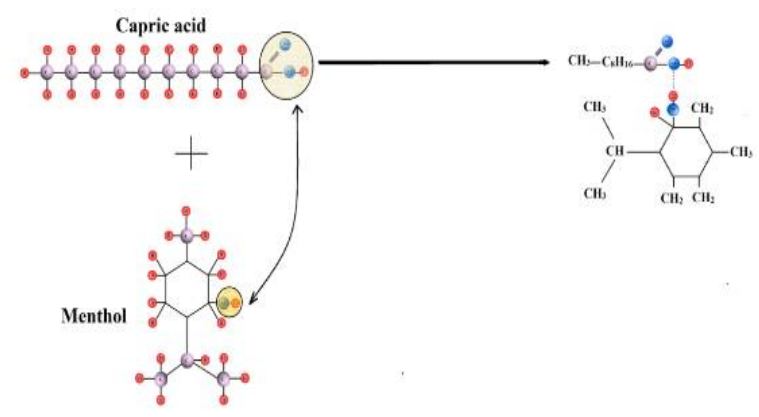

Figure 5: Interaction between the oxygen atom of the hydroxyl group comprising the carboxylic moiety of capric acid by the hydrogen atom of menthol hydroxyl group

Menthol's structure was also influenced by hydrogen bond formation with capric acid. The main $\mathrm{O}-\mathrm{H}$ stretching mode of menthol, centered at $3260 \mathrm{~cm}^{-1}$ (Figure 2), appeared to be spanning a broad transmittance range from $3113 \mathrm{~cm}^{-1}$ to $3392 \mathrm{~cm}^{-1}$ (Figure 4). Such broadening is attributed to a number of overlapping $\mathrm{O}-\mathrm{H}$ stretching peaks ranging from those experiencing strong hydrogen bonding to those of "free" $\mathrm{OH}$ groups. These groups occur at the lowest and 
the highest wavenumbers of the aforementioned range, respectively. Occurrence of different polarities/ or band strengths of the O-H group in this range was attributed to its sensitivity towards hydrogen bond formation [18]. Accordingly, the FTIR of the $\mathrm{O}-\mathrm{H}$ group of menthol-originally located at $3260 \mathrm{~cm}^{-p}$-is strongly evident in the frequencies nearby $3113 \mathrm{~cm}^{-1}$ upon hydrogen bonding with capric acid.

The interaction of the O-H group of menthol with capric acid was further evident by changes in the in-plane and out-of-plane bending vibrations of menthol at 1226 and $668 \mathrm{~cm}^{-1}$ respectively (Figure 2). The shift of these bands to lower frequencies at $1225 \mathrm{~cm}^{-1}$ and $633 \mathrm{~cm}^{-1}$ respectively (Figure 4) suggests increased polarization of the $\mathrm{O}-\mathrm{H}$ group. On the other hand, conformational changes to the menthol structure were manifested by the broadening of the $\mathrm{C}-\mathrm{H}$ frequencies of the methyl groups at 1368 to 1471 $\mathrm{cm}^{-1}$.

Interaction of capric acid with menthol as evident by FTIR was further justified by DSC. The low melting temperature of the complex was earlier attributed to a cis-isomerization structural change whereby a cis-orientation provides poor packing of the crystal lattice. Such behavior weakens the intermolecular forces that strongly hold molecules in the solid state [22]. Accordingly, the poor packing is the reason behind the broadening of the melting peak of the complex. The appearance of less ordered or partially crystalline structures, in general, gives rise to a very broad melting peak [23]. In this regard, the presence of amorphous regions imparts a gradual transition to a melt form. In fact, the formation of eutectic systems was described as a common transformation of crystalline to partially crystalline components [24].

There is another information that can be deduced from the initial exothermic peaks (Figure 7) at $4.26{ }^{\circ} \mathrm{C}$ and $-5.12{ }^{\circ} \mathrm{C}$ and from the drop in the base line at $-7.28^{\circ} \mathrm{C}$. The latter is clear indication of a glass transition state that transferred the frozen solid mixture from glassy to rubbery states as more energy was gained. The former represents the crystallization state which is - in the current case- divided into two peaks corresponding to a two-step process assigned to crystallization and conformational change of the complex [10].

At the 1:4 mass ratio, there is more menthol to establish more hydrogen bonding with capric acid. In contrast, at the 4:1 mass ratio, hydrogen bonding was at its lower extent. Such difference explains the broader melting endotherm at the
1:4 mass compared to the relatively sharp endotherm at the 4:1 mass ratio. Moreover, at the high extent of hydrogen bonding, i.e. at the 1:4 mass ratio, there appears to be another metastable polymorph of the complex. The foregoing showed the first strong sharp melting endotherm at $3.4^{\circ} \mathrm{C}$ overlapping with the second melting endotherm at $15^{\circ} \mathrm{C}$.

The same polymorph further showed a strong sharp crystallization exotherm at $-4.26{ }^{\circ} \mathrm{C}$ which represents the free hydroxyl group as evidenced by the broad FTIR peak at $3260 \mathrm{~cm}^{-1}$. Moreover, at the same ratio (1:4), the hydrogen bonded complex showed weak sharp crystallization exotherm at $-5.12{ }^{\circ} \mathrm{C}$. The appearance of this metastable polymorph was reduced at the 1:1 mass ratio and its DSC response was minimized (Figure 7). In this regard, the first and second melting endotherms were clearly separated, the first crystallization exotherm became weak and quantitatively equivalent to the second crystallization exotherm of the complex. At the 4:1 mass ration, the hydrogen bonded complex was dominant and the metastable polymorph was almost not present. The former was evident by the second melting endotherm which became sharper with minimum broadening and by one crystallization exotherm which became stronger. The later was evident in the very weak melting endotherm and in the disappearance of the first crystallization exotherm.

Such metastable polymorphic form of the capric acid/ menthol complex was visually detected as particles (Figure 10) that disappeared when they underwent complete melting as the temperature was raised above $15^{\circ} \mathrm{C}$.

\section{CONCLUSION}

The work described herein illustrates the formation of eutectic systems between capric acid menthol and addresses two main explanations behind such formation. The initial interaction between the system components is believed to take place via hydrogen bonding between the hydroxyl group oxygen atom of capric acid and the hydrogen atom of menthol hydroxyl group. Such interaction resulted in the transformation of crystalline domains to partially crystalline polymorphic structures. This was attributed to disruption of the crystalline structure of capric acid and menthol into less ordered glassy complexes. Compared to the individual eutectic system components, these complexes are characterized by their lower melting points to an extent that justifies their liquid nature at room temperature. 


\section{DECLARATIONS}

\section{Acknowledgement}

The authors would like to acknowledge the University of Sulaimani/Iraq for their scientific support in addition to the University of Petra/Jordan for providing lab facilities throughout the project.

\section{Conflict of interest}

No conflict of interest is associated with this work.

\section{Contribution of authors}

We declare that this work was done by the authors named in this article and all liabilities pertaining to claims relating to the content of this article will be borne by the authors. Hiba $\mathrm{H}$ Ali and Mowafaq M Ghareeb contributed in the DSC experimental work and discussion. Mayyas AlRemawi and Faisal T Al-Akayleh contributed in the FTIR/microscopy experimental work and discussion.

\section{Open Access}

This is an Open Access article that uses a funding model which does not charge readers or their institutions for access and distributed under the terms of the Creative Commons Attribution License (http://creativecommons.org/licenses/by/ 4.0) and the Budapest Open Access Initiative (http://www.budapestopenaccessinitiative.org/rea d), which permit unrestricted use, distribution, and reproduction in any medium, provided the original work is properly credited.

\section{REFERENCES}

1. Yong CS, Jung SH, Rhee J-D, Choi H-G, Lee B-J, Kim D$C$, Choi YW, Kim C-K. Improved solubility and in vitro dissolution of ibuprofen from poloxamer gel using eutectic mixture with menthol. Drug Delivery 2003; 10 : 179-183.

2. Wang $H$, Meng $F$. The permeability enhancing mechanism of menthol on skin lipids: a molecular dynamics simulation study. J Mol Model 2017; 23: 279.

3. Genc ZK, Canbay CA, Acar SS, Sekerci M, Genc M. Preparation and thermal properties of heterogeneous composite phase change materials based on camphene-palmitic acid. J Therm Anal Calorim 2015; 120: 1679.

4. Ribeiro BD, Florindo CIS, Iff LC, Coelho MA, Marrucho IM. Novel Menthol-based Eutectic Mixtures:
Hydrophobic Low Viscosity Solvents. ACS Sustainable Chem Eng 2015; 3: 2469-2477.

5. Tuntarawongsa S, Phaechamud T. Menthol, Borneol, Camphor and WS-3 Eutectic Mixture. Adv Mat Res 2012; 506: 355-358.

6. Chun-Woong $P$, Heidi M, Tack-Oon O, Ju-Young $K$, Jung-Myung $H$. Phase behavior of itraconazole-phenol mixtures and its pharmaceutical. Int J Pharm 2012; 436: 652-658.

7. Phaechamud T, Tuntarawongsa S, Charoensuksai $P$. Evaporation Behavior and Characterization of Eutectic Solvent and Ibuprofen Eutectic Solution. AAPS PharmSciTech 2016; 17: 1213-1220.

8. Pudney PDA, Mutch KJ, Zhu S. Characterising the phase behaviour of stearic acid and its triethanolamine soap and acid-soap by infrared spectroscopy. Phys Chem Chem Phys 2009; 11: 5010-5018.

9. Shilei L, Neng Z, Guohui F. Eutectic mixtures of capric acid and lauric acid applied in building wallboards for heat energy storage. Energ Buildings 2006; 38: 708711.

10. Kong $W$, Fu $X$, Yuan Y, Liu Z, Lei J. Preparation and thermal properties of crosslinked polyurethane/lauric acid composites as novel form stable phase change materials with a low degree of supercooling. RSC Adv 2017; 7: 29554-29562.

11. Pang SC, Chin SK, Tay SH, Tchong FM. Starchmaleate-polyvinyl alcohol hydrogels with controllable swelling behaviors. Carbohydr Polym 2011; 84: 424429.

12. Vasques CT, Domenech SC, Severgnini VL, Belmonte LAO, Soldi MS, Barreto PLM, Soldi V. Effect of thermal treatment on the stability and structure of maize starch cast films. Starch/Stärke 2007; 59: 161-170.

13. Hadži $D$, Sheppard N. The infra-red absorption bands associated with the $\mathrm{COOH}$ and $\mathrm{COOD}$ groups in dimeric carboxylic acids. I. The region from 1500 to $500 \mathrm{~cm}-1$. Proc R Soc Lond A; 1953; 216: 247-266.

14. Chapman D. The Structure of Lipids. Verlag Methuen \& Co Ltd-London, 1965, chapter 4; 1968.

15. Athokpam B, Ramesh SG, McKenzie RH. Effect of hydrogen bonding on the infrared absorption intensity of $\mathrm{OH}$ stretch vibrations. Chem Phys 2017; 488-489: 4354.

16. Bloch DR. Organic Chemistry: Alpha-Substitution Reactions in Carbonyl Compounds. McGraw-Hill Professional- Second Edition, NY; 2012.

17. Reusch W. Virtual Textbook of Organic Chemistry: Stereoisomers Part I, Michigan State University; revised 2010.

18. Max J-J, Chapados C. Isotope Effects in Liquid Water by Infrared Spectroscopy. III. H2O and D2O Spectra from 6000 to $0 \mathrm{~cm}-1$. J Chem Phys 2009; 131: 184505.

19. Corvis Y, Négrier P, Massip S, Leger J-M, Espeau P. Insights into the crystal structure, polymorphism and thermal behavior of menthol optical isomers and racemates. CrystEngComm 2012; 14: 7055-7064. 
20. Bombicz P, Buschmann J, Luger P, Dung NX, Nam CB. Kristallogr 1999; 214: 423.

21. Turgeon T, Wright L. Mint: The Genus Mentha. J Nat Prod 2007; 70: 1834-1834.

22. Brown RJC, Brown RFC. Melting Point and Molecular Symmetry. J Chem Ed 2000; 77: 724-731.
23. Zhai W, Ko Y, Zhu W, Wong A, Park CB. A study of the crystallization, melting, and foaming behaviors of polylactic acid in compressed $\mathrm{CO}_{2}$. Int $\mathrm{J} \mathrm{Mol} \mathrm{Sci} \mathrm{2009;}$ 10: 5381-5397.

24. Rawlinson CF, Williams AC, Timmins $P$, Grimsey $I$. Polymer mediated disruption of drug crystallinity. Int $J$ Pharm 2007; 336: 42-48. 